\title{
The Future of Learning at the Workplace Is Augmented Reality
}

\author{
Fridolin Wild \\ Oxford Brookes University
}

\begin{abstract}
The next generation of performance support systems and tools for learning at the workplace are likely to be delivered in Augmented Reality. While there are many impressive prototypes and bespoke applications, interoperability has been neglected so far and standards for creating and converting urgently needed AR training content at scale are being missed. To fix this, the IEEE Computer Society is working on a remedy: the working group P1589 is currently creating an AR Learning Experience Model (ARLEM), with a first ballot expected for fall 2016 and a final standard possible as early as summer 2017. The standard in development will provide a data format for the enrichment and exchange of AR learning content, consisting at its core of an activity description language and a workplace model. The standard is built for describing AR learning experiences such as bringing a handbook to life or such as hands-free operator training with smart glasses.
\end{abstract}

Keywords: N. Learning Technologies $\rightarrow$ N.1.g Virtual and augmented reality; H.5.1.b Artificial, augmented, and virtual realities; M.9.2.b Enterprise Performance Management; N.5 Standards and interoperability $\rightarrow$ N.5.a E-learning standards

Experts predict that at least 9 percent of jobs across the globe could be automated. ${ }^{1}$ However, we can avoid big waves of unemployment by promoting next-generation jobs, some perhaps AI assisted. Professionals in such jobs will require new skills, education, and training, but schools and colleges alone can't meet the resultant demand. Clearly, times are calling for new technologies to deliver performance support and learning at the workplace - at scale.

Augmented reality (AR) is extremely effective in increasing worker learning and accuracy by delivering performance guides for learning and performing on the job. ${ }^{2}$ Conducting an activity hands on while being instructed boosts a learner's ability to retain knowledge. This translates to lower costs to train employees and to improve performance. However, the lack of AR interoperability and, consequently, AR content stands in the way of rolling out such training to an emerging market in the numbers required.

The IEEE Computer Society is working on a remedy; working group P1589 is developing an AR Learning Experience Model (ARLEM). A first ballot is planned for fall 2016, and the final standard could be ready as early as summer 2017 .

\section{ARLEM APPLICATIONS}

The emerging specification, a data format for the enrichment and exchange of AR learning content, consists at its core of an activity description language and a workplace model. The XML activity description language lets software vendors of training applications script AR live guidance through workflows, thereby referencing objects and other real-world characteristics of the training environment described in the XML workplace model.

Although current AR applications focus on supporting engineering activities on the shop floor, they could be used in many other areas, such as building and maintaining highly complex enterprise IT infrastructures.

Data needed for developing workplace models for AR learning experiences is usually distributed across a multitude of systems in the enterprise. For example, computer-aided design or engineering tools, product lifecycle management systems, customer-relationship management suites, and electronic product catalogues often hold data and media assets required for the production of high-quality AR content. 
ARLEM data might be used to bring a handbook to life on a tablet computer (see Figure 1). For example, animations of which screws to take out in order to remove a part are visualised in 3D. Animation is a powerful tool and allows much shorter instruction time than verbally describing what to do. This reduces time to mastery as well as saves effort in translation to different languages.

Figure 1. Handbook brought to life on a tablet computer. Superimposed over the helicopter illustration, a 3D-animation guides the user step by step through the procedure of how to change the tail-rotor blade. Additional instructional material (such as explanatory audio/video) can further enhance the learning activity where needed and link in existing multimedia training material.

ARLEM data can also describe experiences for Smart Glasses, as depicted in Figure 2. With the appropriate activity and workplace definition available, the Smart Glasses app can overlay symbols for instruction and then provide feedback on the user's actions. Seeing what needs to be done, step by step, helps guide users through a hands-free practice, providing underlying knowledge and explanations in the context where they're most urgently needed and likely to be remembered. Smart Glasses also can obviate the onus of having to glance back and forth between instructions and the physical items being manipulated.

Figure 2. "Holographic" instruction overlaid on actual object using augmented reality Smart Glasses. A potentiometer on the smart object is overlaid with an animated glyph instructing the user to crank up the voltage, while providing a real-time reading of the current voltage value read directly from the smart object with Internet-of-Things protocols.

In Figure 2, a potentiometer on the smart object is overlaid with an animated glyph instructing the user to crank up the voltage, while providing a real-time reading of the current voltage value read directly from the smart object with Internet-of-Things protocols.

The separation of activity scripts from the workplace model is justified by the frequency of updates required. Although activities might change rather quickly, information about tools, machines, and the general physicality of the workplace requires much less frequent updating. A single workplace model can be referenced in a multitude of learning and training activities performed in that same space and could be used in future performance augmentation systems.

\section{ARLEM SCRIPTS}

Activity scripts conceptually follow the sequencing of a workflow's action steps by specifying, for each action step, which visual augmentation to activate and deactivate when entering and exiting. Figure 3 provides an example of such activity script. The first action step, launched when loading the activity, directs a downward pointing animated arrow to appear with a label "Open here" when the object called "panel" (see script) is encountered. When the user removes the panel, the underlying compartment becomes visible and triggers the launch of the next action step. There, after removal of the arrow and label from the previous step in its exit loop, another animated visual symbol is displayed over the battery in the compartment, indicating that the user should remove it by lifting it out.

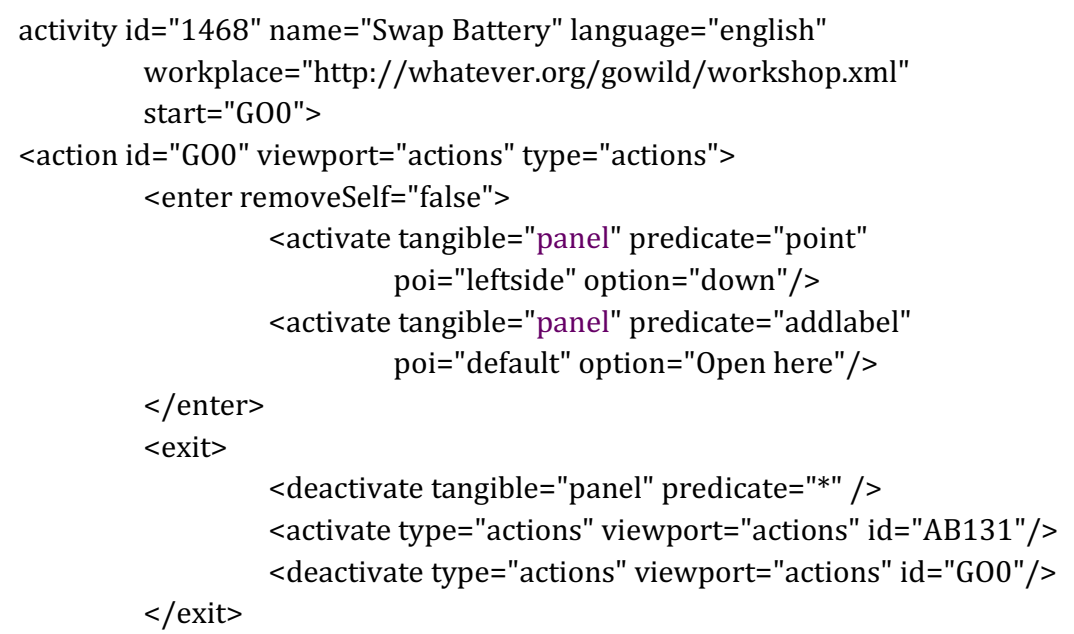




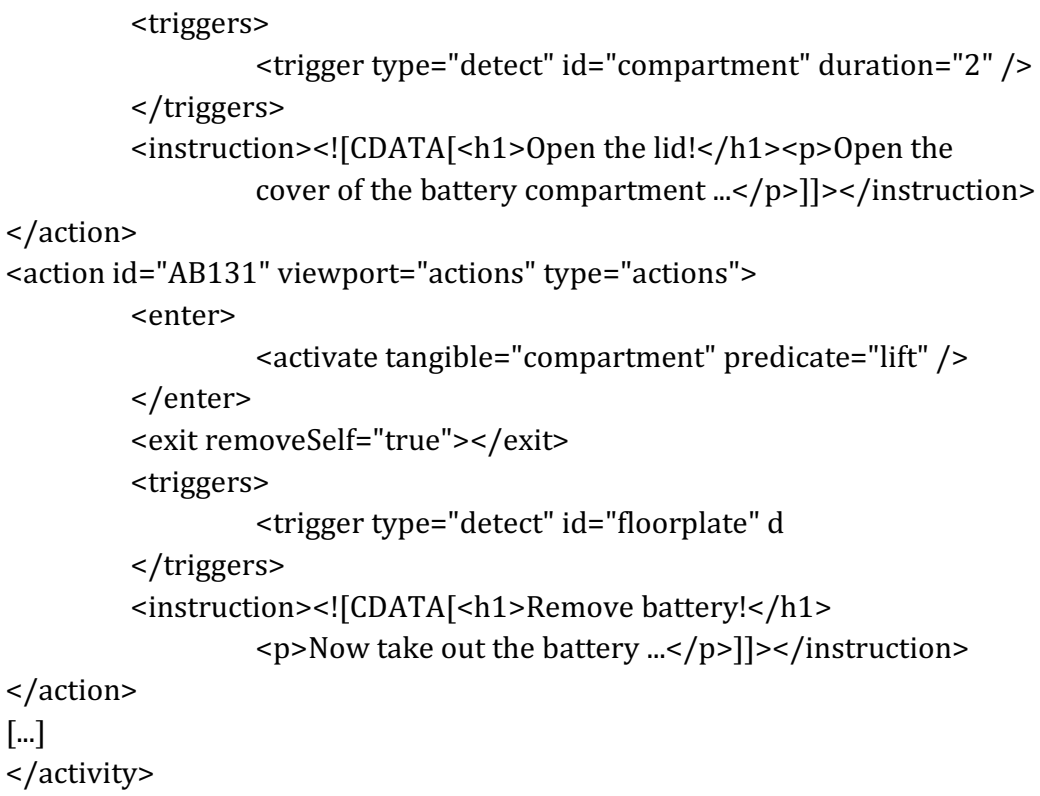

Figure 3. Example activity script for changing a battery. The first action step, launched when loading the activity, directs a downward pointing animated arrow to appear with a label "Open here" when the object named 'panel' is encountered.

To be accessible in both action steps, the two tangible objects of the real world (panel and compartment) must be defined in the workplace model, including links to the data packages for the AR computer vision system used to detect and track objects. Moreover, the workplace model holds a predefined standard vocabulary of handling and movement verbs, including the graphic files for visualization.

Just as HTML markup language uses headlines, paragraphs, and links to describe webpages, ARLEM uses actions, things, and triggers to describe interactions with the real world.

The choice for XML as the representation format wasn't random. The use of XML ensures both human and machine readability. Moreover, many existing technical documentation standards like MuMaSy or DITA (www.oasis-open.org/committees/dita) are based on XML (and Standard Generalized Markup Language), thus allowing the use of standard transformation instruments to upgrade existing content. Other bindings (for instance, to JavaScript Object Notation) might follow.

The emerging AR apps ecosystem requires interchange formats to foster innovation and lower development costs, while providing investment stability for the enterprise and avoiding vendor lock-in.

Predicting the future isn't easy. In most cases, any forecast will be wrong. It is, however, not too farfetched to believe that with AR and ARLEM, mastering new jobs will become easier, cheaper, and faster, and the future of learning and training at the workplace will have a little more magic.

\section{References}

1. M. Arntz, T. Gregory, and U. Zierahn, "The Risk of Automation for Jobs in OECD Countries," OECD Social, Employment and Migration Working Papers, no. 189, 14 May 2016; www.oecd-ilibrary.org/socialissues-migration-health/the-risk-of-automation-for-jobs-in-oecd-countries_5jlz9h56dvq7-en.

2. A. George, "Lockheed Is Using These Augmented Reality Glasses to Build Fighter Jets," Popular Mechanics, 6 Feb. 2015; www.popularmechanics.com/flight/a13967/lockheed-martin-augmented-reality-f35.

Fridolin Wild is a Senior Research Fellow leading the Performance Augmentation Lab at Oxford Brookes University, part of the Department of Computing and Communications Technologies. Contact him atwild@brookes.ac.uk. 